\title{
Reciprocity calibration of Distributed Massive MIMO Access Points for Coherent Operation
}

J oao Vieira and Erik G. Larsson

The self-archived postprint version of this conference paper is available at Linköping University Institutional Repository (DiVA):

http:// urn.kb.se/ resolve?urn=urn:nbn:se:liu:diva-181826

N.B.: When citing this work, cite the original publication.

Vieira, J., Larsson, E. G., (2021), Reciprocity calibration of Distributed Massive MIMO Access Points for Coherent Operation, 2021 IEEE 32nd Annual International Symposium on Personal, Indoor and Mobile Radio Communications (PIMRC), 783-787.

https:// doi.org/ 10.1109/PIMRC50174.2021.9569495

Original publication available at:

https:// doi.org/ 10.1109/ PIMRC50174.2021.9569495

Copyright: IEEE

http://www.ieee.org/

(C) 2021 IEEE. Personal use of this material is permitted. However, permission to reprint/republish this material for advertising or promotional purposes or for creating new collective works for resale or redistribution to servers or lists, or to reuse any copyrighted component of this work in other works must be obtained from the IEEE. 


\title{
Reciprocity calibration of Distributed Massive MIMO Access Points for Coherent Operation
}

\author{
Joao Vieira, Erik G. Larsson \\ Ericsson Research, joao.vieira@ericsson.com \\ Linköping University, erik.g.larsson@liu.se
}

\begin{abstract}
Novel network architectures for 6G distributed massive MIMO systems rely on coherent signaling by distributed antenna panels which are coordinated by a central controller. This type of network architecture is based on reciprocity operation where antenna panels rely on uplink channel estimates for coherent downlink precoding. This paper proposes a calibration method for distributed massive MIMO systems, which overcomes hardware non-reciprocities in order to enable reciprocity-based operation. Measurements for system calibration are collected via a beam-sweep between all pairs of antenna panels. We lay out the system model for this new setup, and propose a maximum likelihood-based procedure to compute calibration coefficients based on the collected measurement set. The procedure is computationally efficient and stable, since 1) each iteration has a closed-form, and 2) the procedure is guaranteed to converge to at least a local optimum (or saddle point). Simulations indicate significant calibration improvements compared to re-using state of the art calibration schemes for the problem at hand.
\end{abstract}

Keywords-6G, cell-free MIMO, Distributed massive MIMO, antenna arrays, antenna panels, reciprocity calibration, channel reciprocity.

\section{INTRODUCTION}

$\mathrm{D}$ istributed Massive MIMO, also known as cell-free massive MIMO, is an emerging technology and a candidate for $6 \mathrm{G}$ multi-antenna systems [1]. It relies on phase-coherent operation of large numbers of antenna panels, which are distributed over a larger area. Two important implementations are RadioStripes (with antennas along a cable) and large intelligent surfaces, e.g. RadioWeaves [2] (with antennas integrated into walls and other objects). It is foreseen that practical distributed massive MIMO systems will be built of access points (APs), and that each AP comprise multiple antennas elements (creating a, so-called, antenna panel), and where the APs are interconnected with one another via a central processing unit (CPU). The preferred operation is in time-division duplexing mode, where uplink-downlink reciprocity of the radio channel can be exploited in order to estimate the downlink channels using uplink pilots, much like in conventional massive MIMO. This operation mode is typically referred to as reciprocity-based operation.

However, the assumption of uplink-downlink radio channel reciprocity is only true in practice to a certain extent. More specifically, even though it is well known that the propagation channel is reciprocal, the transceiver radio frequency (RF) units sitting at both ends of the radio link are generally not.
Thus, transceiver calibration is required in order to be possible for the system to operate in reciprocity-based mode.

There exist different types of calibration approaches in the literature that restore some degree of reciprocity of a wireless link. Some approaches rely on 1) bi-directional signaling between user equipments (UEs) and the base station (BS), other approaches rely on 2) internal calibration cable networks at the base-station end of the link, and other approaches rely on 3 ) over-the-air signaling between pairs of antennas of the base station. However, for the setup of a distributed massive MIMO systems, only the third approach is suitable since the system should typically not rely on the quality of the link to UEs for calibration, nor is it suitable to install cables between distributed APs with the sole purpose of system calibration. Several works in this vein for are [4,5].

However, the calibration approaches in the literature address system setups where each transceiver to be calibrated is associated with a single antenna element, i.e. they address calibration of fully-digital systems. In addition, it is foreseen that practical distributed massive MIMO systems will be built of multiple APs, and that each AP comprise multiple antennas elements but only a few transceiver chains. Such AP's multiple antennas are controlled via (tunable) analog beamforming, mainly implemented in the RF stage of the radio. The key insight for distributed massive MIMO calibration is that the possibility of properly tuning, and hence sweeping, such beamformers provide extra degrees of freedom to the system which can be exploited to obtain a multitude of over-the-air measurements for calibration purposes.

To the authors' current knowledge, there is no prior art proposing calibration methods for said type of distributed massive MIMO system setups.

\section{A. Main Contributions of the Paper}

In this paper, we study reciprocity calibration of distributed massive MIMO systems. In particular, we write a system model for the calibration setup, where joint beam scanning procedure is executed by all APs of the network in order to collect measurements for calibration. We lay out the maximum-likelihood (ML) cost function for the calibration problem at hand, and propose a computationally-efficient alternating optimization procedure. We show that the optimization procedure is guaranteed to converge. We perform simulations for the current system setup, and verify performance enhancements of the proposed method, compared to straightforward applications of state-of-the-art calibration schemes in the context of distributed massive MIMO. 


\section{SIGNAL MODEL}

\section{A. Problem Description (prior art $[3,4]$ )}

For illustrative but non-limiting purposes, assume a narrowband distributed massive MIMO link with $K$ singleantenna UEs on the other end, and $M$ transceiver chains at the other end, e.g., assume that each transceiver chain is associated with a distinct AP. Assuming a noiseless channel for the moment, the $M \times K$ uplink narrowband radio channel $\boldsymbol{H}_{U L}$, representing e.g. an orthogonal frequency division multiplexing (OFDM) subcarrier, is modelled as

$$
\boldsymbol{H}_{U L}=\boldsymbol{R}_{A P} \boldsymbol{H} \boldsymbol{T}_{U E}
$$

where $\boldsymbol{H}$ is a matrix comprising all channels effects occurring between the transmitter and receiver chains. For example, in fully-digital beamforming systems, the channel matrix $\boldsymbol{H}$ typically denotes the propagation channel [3]. The matrix $\boldsymbol{T}_{U E}=\operatorname{diag}\left\{t_{1}^{U E}, \ldots, t_{K}^{U E}\right\}$ is a diagonal matrix where each diagonal entry models the complex gain of each UE's transmitter chain, and $\boldsymbol{R}_{A P}=\operatorname{diag}\left\{r_{1}^{A P}, \ldots, r_{M}^{A P}\right\}$ is a diagonal matrix where each diagonal entry models the complex gain of each AP's receiver chain.

Within the same time/frequency coherence interval, the associated downlink channel is given by

$$
\boldsymbol{H}_{D L}=\boldsymbol{R}_{U E} \boldsymbol{H}^{T} \boldsymbol{T}_{A P}
$$

where $(.)^{T}$ denotes the transpose operator, and $\boldsymbol{T}_{A P}=$ $\operatorname{diag}\left\{t_{1}^{A P}, \ldots, t_{M}^{A P}\right\}$ and $\boldsymbol{R}_{U E}=\operatorname{diag}\left\{r_{1}^{U E}, \ldots, r_{K}^{U E}\right\}$ model the associated transmitter and receiver gains of each UE and each $\mathrm{AP}$, respectively.

Note that the channel matrix $\boldsymbol{H}$ is assumed to be reciprocal. However, the end-to-end radio channel is not reciprocal, i.e. $\boldsymbol{H}_{U L} \neq \boldsymbol{H}_{D L}^{T}$. This is because the gains of the transceiver circuitries are not reciprocal (e.g., $\boldsymbol{T}_{A P} \neq \boldsymbol{T}_{U E}$ ). Due to this non-reciprocity aspect, it is not immediately obvious how downlink transmission can be performed based on channel estimates obtained from uplink pilot signals.

To indicate how to address the challenge with the nonreciprocal transceiver terms, let's assume for now that the AP side of the link has knowledge of the following matrix

$$
\boldsymbol{C}=\operatorname{diag}\left\{c_{1}, \ldots, c_{M}\right\}=\boldsymbol{T}_{A P}\left(\boldsymbol{R}_{A P}\right)^{-1},
$$

up to an a non-zero complex-valued unknown scaling term $\alpha$.

Via uplink pilot signals, the BS can estimate $\boldsymbol{H}_{U L}$. If the APs wants to jointly perform, e.g. ZF transmission to the UEs, they do so by the computing the Moore-Penrose inverse of $\boldsymbol{H}_{U L}^{T}$, namely

$$
\boldsymbol{P}=\boldsymbol{H}_{U L}^{*}\left(\boldsymbol{H}_{U L}^{T} \boldsymbol{H}_{U L}^{*}\right)^{-1}
$$

where ( $)^{*}$ denotes element-wise complex conjugation. However, since the matrix $\boldsymbol{P}$ was computed via uplink signals, it cannot be directly used as a downlink precoder since it is not matched to the (non-reciprocal) downlink channel $\boldsymbol{H}_{D L}$. To solve this, each AP multiplies its pre-coded signals with its associated entry of $(\alpha \boldsymbol{C})^{-1}$. More specifically, the pre-coded signal at transceiver $m$ is multiplied with $c_{m}$, with $1 \leq m \leq M$. With that, the effective downlink channel is written as

$$
\begin{aligned}
& \boldsymbol{H}^{\prime}{ }_{D L} \\
& =\boldsymbol{H}_{D L} \alpha^{-1} \boldsymbol{C}^{-1} \boldsymbol{P} \\
& =\boldsymbol{H}_{D L} \alpha^{-1} \boldsymbol{C}^{-1} \boldsymbol{H}_{U L}^{*}\left(\boldsymbol{H}_{U L}^{T} \boldsymbol{H}_{U L}^{*}\right)^{-1} \\
& =\boldsymbol{H}_{D L} \alpha^{-1} \boldsymbol{C}^{-1} \boldsymbol{R}_{A P}^{*} \boldsymbol{H}^{*} \boldsymbol{T}_{U E}^{*}\left(\boldsymbol{T}_{U E} \boldsymbol{H}^{T}\left|\boldsymbol{R}_{A P}\right|^{2} \boldsymbol{H}^{*} \boldsymbol{T}_{U E}^{*}\right)^{-1} \\
& =\boldsymbol{H}_{D L} \alpha^{-1} \boldsymbol{T}_{A P}^{-1}\left|\boldsymbol{R}_{A P}\right|^{2} \boldsymbol{H}^{*} \boldsymbol{T}_{U E}^{*}\left(\boldsymbol{T}_{U E} \boldsymbol{H}^{T}\left|\boldsymbol{R}_{A P}\right|^{2} \boldsymbol{H}^{*} \boldsymbol{T}_{U E}^{*}\right)^{-1} \\
& \quad=\boldsymbol{H}_{D L} \alpha^{-1}\left(\boldsymbol{T}_{A P}\right)^{-1}\left(\boldsymbol{H}^{T}\right)^{\dagger}\left(\boldsymbol{T}_{U E}\right)^{-1} \\
& =\alpha^{-1} \boldsymbol{R}_{U E} \boldsymbol{H}^{T} \boldsymbol{T}_{A P}\left(\boldsymbol{T}_{A P}\right)^{-1}\left(\boldsymbol{H}^{T}\right)^{\dagger}\left(\boldsymbol{T}_{U E}\right)^{-1} \\
& =\alpha^{-1} \boldsymbol{R}_{U E}\left(\boldsymbol{T}_{U E}\right)^{-1},
\end{aligned}
$$

which is a diagonal channel matrix with unknown diagonal entries. The operator $(.)^{\dagger}$ denotes the Moore-Penrose inverse, and $|.|^{2}$ denotes element-wise squared absolute value.

Such unknown diagonal entries can be obtained using only one downlink reference signal, which is beamformed in the downlink towards all UEs, using the calibrated channels. Thus, $K$ uplink pilot signals plus one downlink reference signal are sufficient to conduct all training needed for this type of calibrated reciprocity-based transmissions. This results in much less training overhead compared to explicit downlink channel estimation.

In conclusion, knowledge of the matrix $\boldsymbol{C}$ allows coherent transmissions, e.g. ZF downlink transmissions, with no interuser interference over what is effectively a calibrated uplink/downlink channel setup. The diagonal matrix $\boldsymbol{C}$ can thus be interpreted as a calibration matrix, and thus we are interested to estimate its diagonal elements.

\section{B. Calibration Signal Model}

In this sub-section we propose our method to estimate the calibration matrix $\boldsymbol{C}$.

We note that the novelty of our proposal lies in the fact that the effective channel $\boldsymbol{H}$ in (1) is not only made of the propagation channel, as in prior calibration works in massive MIMO, but is also comprised by a beamforming matrix $\boldsymbol{W}$. More specifically

$$
\boldsymbol{H}=\boldsymbol{W} \boldsymbol{H}_{p}
$$

where $\boldsymbol{H}_{p}$ models the propagation uplink channel and each AP's beamformer is given by each row of $\boldsymbol{W}$. The possibility of 
tunning such beamformers provide extra degrees of freedom to the system which can be exploited to obtain a multitude of measurements for calibration purposes. We elaborate on this concept in the current and next sub-sections.

Our measurement procedure for calibration, relies of a joint beam sweep by all network APs. That is, an AP acts as a transmitter and scans each of its $L$ beams while the other $M-$ 1 APs act as receivers and scan each of their $L$ beams as well. (For simplicity, assume that all transmitters and receivers are capable of grid-of-beams beamforming, and that $L$ denotes the number of transmit and receive beams at all APs). This process is repeated with another AP acting as transmitter, and it stops when all APs have as transmitters. The number of measurements resulting from this extensive beam sweeping procedure is therefore $M L^{2}$. (We note that, other sub-cases where a partial beam scanning is done are also possible, but we keep the formulation to be the one of a full-beam scanning in the paper since it represents the general case.)

Set $r_{i} \triangleq r_{i}^{A P}$ and $t_{i} \triangleq t_{i}^{A P}$ for notation convenience. With that, the signals collected by a receiving $\mathrm{AP}$, say $\mathrm{AP} j$ with $1 \leq j \leq$ $M$, after another AP, say $\mathrm{AP} i$ with $1 \leq i \leq M$ and $i \neq j$, scanned its beam, can be written as

$$
\boldsymbol{Y}_{i, j}=r_{i} \boldsymbol{F}_{i}^{T} \boldsymbol{H}_{p}^{i, j} \boldsymbol{B}_{j} t_{j}+\boldsymbol{N}_{i, j}
$$

where $\boldsymbol{N}_{i, j}$ is a matrix modelling receive noise and its entries are independent and identically distributed zero-mean circularly symmetric complex-valued Gaussian variables with variance $\sigma^{2}$, and the symmetric matrix $\boldsymbol{H}_{p}^{i, j}$ denotes the reciprocal propagation channel from all antenna elements of AP $j$ to all antenna elements of $\mathrm{AP} i$. The matrix $\boldsymbol{B}_{j}=$ $\left[\boldsymbol{b}_{j}(1) \ldots \boldsymbol{b}_{j}(L)\right]$ denotes the transmit codebook matrix at AP $j$ and contains all possible $L$ beams of the transmit beamformer. Similarly, $\boldsymbol{F}_{i}=\left[\boldsymbol{f}_{i}(1) \ldots \boldsymbol{f}_{i}(L)\right]$ denotes the receive codebook matrix which contains all possible beams of the receive beamformer. (Note the relation between $\boldsymbol{W}$ in (4), and the codebook matrices in (5), e.g. $\boldsymbol{B}_{j}$. To explain, the $j$ th row of $\boldsymbol{W}$ consists of the beam being used by the $j$ th AP for downlink data transmission, which is a given column of the transmit grid-ofbeams matrix $\boldsymbol{B}_{j}$ ). The matrix $\boldsymbol{Y}_{i, j}$ contains the measurements resulting from all combinations of beam pairs.

Based on (5), the vectorized signal model is

$$
\begin{array}{rlr}
\operatorname{vec}\left(\mathbf{Y}_{i, j}\right)= & \operatorname{vec}\left(r_{i} \boldsymbol{F}_{i}^{T} \boldsymbol{H}_{p}^{i, j} \boldsymbol{B}_{j} t_{j}\right) \\
& +\operatorname{vec}\left(\mathbf{N}_{i, j}\right) & \\
= & \operatorname{vec}\left(r_{i} r_{j} \boldsymbol{F}_{i}^{T} \boldsymbol{H}_{p}^{i, j} \boldsymbol{B}_{j} \frac{t_{j}}{r_{j}}\right) & +\operatorname{vec}\left(\mathbf{N}_{i, j}\right) \\
= & r_{i} r_{j} \operatorname{vec}\left(\boldsymbol{F}_{i}^{T} \boldsymbol{H}_{p}^{i, j} \boldsymbol{B}_{j}\right) c_{j} & +\operatorname{vec}\left(\mathbf{N}_{i, j}\right) \\
= & r_{i} r_{j}\left[\begin{array}{ccc}
\boldsymbol{F}_{i}^{T} \boldsymbol{H}_{p}^{i, j} & \mathbf{0} & \mathbf{0} \\
\mathbf{0} & \ddots & \mathbf{0} \\
\mathbf{0} & \mathbf{0} & \boldsymbol{F}_{i}^{T} \boldsymbol{H}_{p}^{i, j}
\end{array}\right] \operatorname{vec}\left(\boldsymbol{B}_{j}\right) c_{j} \\
& \quad+\operatorname{vec}\left(\mathbf{N}_{i, j}\right)
\end{array}
$$

where the operator $\operatorname{vec}($.$) stacks the columns of its matrix$ input.
Each AP uses the same beams for transmission and reception, e.g., $\boldsymbol{B}_{i}=\boldsymbol{F}_{i}$ and $\boldsymbol{B}_{j}=\boldsymbol{F}_{j}-$ this assumption is motivated later on. We also write the reciprocal propagation matrix as $\boldsymbol{H}_{p} \triangleq \boldsymbol{H}_{p}^{i, j}=\left(\boldsymbol{H}_{p}^{j, i}\right)^{T}$ for simplicity. With that, it follows that the double-directional signal model between AP $i$ and $\mathrm{AP} j$, can be written as

$$
\begin{aligned}
& \overline{\boldsymbol{Y}}_{i, j}=\left[\begin{array}{l}
\operatorname{vec}\left(\boldsymbol{Y}_{i, j}\right) \\
\operatorname{vec}\left(\boldsymbol{Y}_{j, i}^{T}\right)
\end{array}\right] \\
& =\left[\begin{array}{c}
r_{i} r_{j}\left[\begin{array}{ccc}
\boldsymbol{F}_{i}^{T} \boldsymbol{H}_{p}^{i, j} & \mathbf{0} & \mathbf{0} \\
\mathbf{0} & \ddots & \mathbf{0} \\
\mathbf{0} & \mathbf{0} & \boldsymbol{F}_{i}^{T} \boldsymbol{H}_{p}^{i, j}
\end{array}\right] \operatorname{vec}\left(\boldsymbol{B}_{j}\right) c_{j} \\
r_{j} r_{i}\left[\begin{array}{ccc}
\boldsymbol{F}_{i}^{T} \boldsymbol{H}_{p}^{i, j} & \mathbf{0} & \mathbf{0} \\
\mathbf{0} & \ddots & \mathbf{0} \\
\mathbf{0} & \mathbf{0} & \boldsymbol{F}_{i}^{T} \boldsymbol{H}_{p}^{i, j}
\end{array}\right] \operatorname{vec}\left(\boldsymbol{B}_{j}\right) c_{i}
\end{array}\right] \\
& +\left[\begin{array}{l}
\operatorname{vec}\left(\boldsymbol{N}_{i, j}\right) \\
\operatorname{vec}\left(\boldsymbol{N}_{j, i}^{T}\right)
\end{array}\right] \\
& =\left[\begin{array}{cc}
\boldsymbol{e}_{i, j} & \mathbf{0} \\
\mathbf{0} & \boldsymbol{e}_{i, j}
\end{array}\right]\left[\begin{array}{l}
c_{j} \\
c_{i}
\end{array}\right]+\left[\begin{array}{l}
\operatorname{vec}\left(\boldsymbol{N}_{i, j}\right) \\
\operatorname{vec}\left(\boldsymbol{N}_{j, i}^{T}\right)
\end{array}\right] \\
& =\left[\begin{array}{cc}
\boldsymbol{e}_{i, j} & \mathbf{0} \\
\mathbf{0} & \boldsymbol{e}_{i, j}
\end{array}\right]\left[\begin{array}{l}
c_{j} \\
c_{i}
\end{array}\right] \quad+\overline{\boldsymbol{N}}_{i, j}
\end{aligned}
$$

Based on the bi-directional model obtained in (6), reciprocity calibration of a multiple APs can be carried out.

We note that the transmit and receive codebooks, namely $\boldsymbol{B}_{j}$ and $\boldsymbol{F}_{i}$, may be tall matrices (i.e. with more rows than columns) or square matrices (e.g. DFT matrices). The exact setting of such codebooks is not needed to be known by the system to perform calibration. The only assumption required is that the same AP beams are used for transmission and reception (i.e. $\boldsymbol{B}_{i}=\boldsymbol{F}_{i}$ ), otherwise $\boldsymbol{e}_{i, j} \neq \boldsymbol{e}_{j, i}$ and (6) may not be identifiable.

\section{Maximum-Likelihood Calibration of a 3-AP network}

Next, we exemplify the case where $K=3$ APs are being jointly calibrated for reciprocity - the generality of the approach scales for any number of APs. With that, the joint bi-directional signal model may be written as

$$
\overline{\boldsymbol{Y}}=\boldsymbol{C}_{e q}\left[\begin{array}{l}
\boldsymbol{e}_{1,2} \\
\boldsymbol{e}_{1,3} \\
\boldsymbol{e}_{2,3}
\end{array}\right]+\overline{\boldsymbol{N}}
$$

or

$$
\overline{\bar{Y}}=E_{e q} c \quad+\overline{\bar{N}}
$$

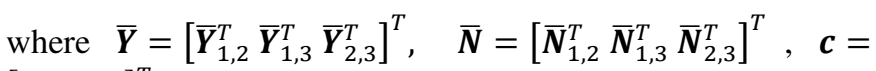
$\left[\begin{array}{lll}c_{1} & c_{2} & c_{3}\end{array}\right]^{T}$, and 


$$
\boldsymbol{E}_{e q}=\left[\begin{array}{ccc}
\boldsymbol{e}_{1,2} & \mathbf{0} & \mathbf{0} \\
\boldsymbol{e}_{1,3} & \mathbf{0} & \mathbf{0} \\
\mathbf{0} & \boldsymbol{e}_{1,2} & \mathbf{0} \\
\mathbf{0} & \boldsymbol{e}_{2,3} & \mathbf{0} \\
\mathbf{0} & \mathbf{0} & \boldsymbol{e}_{1,3} \\
\mathbf{0} & \mathbf{0} & \boldsymbol{e}_{2,3}
\end{array}\right]
$$

and

$$
\boldsymbol{C}_{e q}=\left[\begin{array}{ccc}
\operatorname{diag}\left(\mathrm{c}_{1}, \ldots c_{1}\right) & \mathbf{0} & \mathbf{0} \\
\operatorname{diag}\left(\mathrm{c}_{2}, \ldots c_{2}\right) & \mathbf{0} & \mathbf{0} \\
\mathbf{0} & \operatorname{diag}\left(\mathrm{c}_{1}, \ldots c_{1}\right) & \mathbf{0} \\
\mathbf{0} & \operatorname{diag}\left(\mathrm{c}_{3}, \ldots c_{3}\right) & \mathbf{0} \\
\mathbf{0} & \mathbf{0} & \operatorname{diag}\left(\mathrm{c}_{2}, \ldots c_{2}\right) \\
\mathbf{0} & \mathbf{0} & \operatorname{diag}\left(\mathrm{c}_{3}, \ldots c_{3}\right)
\end{array}\right] .
$$

Note that $\overline{\overline{\boldsymbol{Y}}}$ and $\overline{\overline{\boldsymbol{N}}}$ are matrices with the same dimensions of $\overline{\boldsymbol{Y}}$ and $\overline{\boldsymbol{N}}$, but with elements re-ordered. The re-ordering can be inferred from the structure of $\boldsymbol{E}_{e q}$ and $\boldsymbol{c}$ in (8).

To estimate the calibration coefficients $\boldsymbol{c}$, a maximum likelihood cost function can be written. From an optimization point of view, it is equivalent to minimizing the squared residuals, namely,

$$
J_{M L}\left(\boldsymbol{E}_{e q}, \boldsymbol{c}\right)=\left\|\overline{\boldsymbol{Y}}-\boldsymbol{E}_{e q} \boldsymbol{c}\right\|^{2}
$$

where the operator $\|\boldsymbol{A}\|^{2}$ denotes the squared Frobenious norm of matrix $\boldsymbol{A}$.

\section{An Alternating Optimization Procedure}

Our task now is to find the values of the calibration coefficients $\boldsymbol{c}$ and the nuisance matrix $\boldsymbol{E}_{e q}$ that minimize $J_{M L}\left(\boldsymbol{E}_{e q}, \boldsymbol{c}\right)$. Note that there is no obvious closed-form solution for $\boldsymbol{E}_{e q}$ and $\boldsymbol{c}$ since the cost function is not quadratic on the joint parameter space.

However, by fixing one part of the parameter space, e.g. $\boldsymbol{E}_{e q}$, a closed-form solution exists for the remaining part of the parameter space, e.g. $\boldsymbol{c}$, and it can trivially be obtained can be obtained via

$$
\hat{\boldsymbol{c}}=\boldsymbol{E}_{e q}^{\dagger} \overline{\boldsymbol{Y}}
$$

where $\boldsymbol{A}^{\dagger}$ denotes the Moore-Penrose inverse of $\boldsymbol{A}$. With that, one can use alternative optimization techniques to find suitable estimates for $\boldsymbol{E}_{e q}$ and $\boldsymbol{c}$. More specifically, letting the estimates obtained at the $n^{\text {th }}$ iteration of said alternating procedure be given by $\widehat{\boldsymbol{c}}^{(n)}$ and $\widehat{\boldsymbol{E}}_{e q}^{(n)}$, the estimates at iteration $n+1$ may be obtained via the 2 consecutive steps

$$
\widehat{\boldsymbol{c}}^{(n+1)}=\left(\widehat{\boldsymbol{E}}_{e q}^{(n)}\right)^{\dagger} \overline{\boldsymbol{Y}}
$$

and

$$
\widehat{\boldsymbol{E}}_{e q}^{(n+1)}=\widehat{\boldsymbol{C}}_{e q}^{(n+1)} \overline{\boldsymbol{Y}}
$$

where $\widehat{\boldsymbol{C}}_{e q}^{(n+1)}$ consist of the matrix $\boldsymbol{C}_{e q}$ with the estimates $\widehat{\boldsymbol{c}}^{(n+1)}$ in its entries.

\section{E. Convergence of the Optimization Procedure}

The main aspect to highlight is that the optimization procedure described above is guaranteed to converge either to the global optimum, a local optimum or a saddle point of $J_{M L}\left(\boldsymbol{E}_{e q}, \boldsymbol{c}\right)$. A simple proof follows.

Prior to iteration $n+1$, inserting the current estimates $\widehat{\boldsymbol{c}}^{(n)}$ and $\widehat{\boldsymbol{E}}_{e q}^{(n)}$ in the cost function, will yield an associated cost of, say, $C_{(n, n)}$. Mathematically,

$$
J_{M L}\left(\widehat{\boldsymbol{E}}_{e q}^{(n)}, \widehat{\boldsymbol{c}}^{(n)}\right)=C_{(n, n)} .
$$

Note that, in general, $\widehat{\boldsymbol{c}}^{(n)}$ is not optimized for the current value of $\widehat{\boldsymbol{E}}_{e q}^{(n)}$. This is true if, e.g., both $\widehat{\boldsymbol{c}}^{(n)}$ and $\widehat{\boldsymbol{E}}_{e q}^{(n)}$ are randomly initialized which can be the case when $n=0$, or if $\widehat{\boldsymbol{c}}^{(n)}$ and $\widehat{\boldsymbol{E}}_{e q}^{(n)}$ are obtained via iteration $n$ the proposed alternating optimization procedure.

The first step of iteration $n+1$ of the proposed procedure, optimizes $\hat{\boldsymbol{c}}$ while having $\widehat{\boldsymbol{E}}_{e q}$ fixed, namely, fixed to its current value is $\widehat{\boldsymbol{E}}_{e q}^{(n)}$. Since this optimization exists in closed form, we are guaranteed to find the optimum (i.e. minimum) value of $\widehat{\boldsymbol{c}}$ for that particular $\widehat{\boldsymbol{E}}_{e q}^{(n)}$. More specifically,

$$
J_{M L}\left(\widehat{\boldsymbol{E}}_{e q}^{(n)}, \widehat{\boldsymbol{c}}^{(n+1)}\right) \leq J_{M L}\left(\widehat{\boldsymbol{E}}_{e q}^{(n)}, \widehat{\boldsymbol{c}}\right), \forall \hat{\boldsymbol{c}} .
$$

and thus the associated cost function value does not increase compared to (10). With that, we have

$$
J_{M L}\left(\widehat{\boldsymbol{E}}_{e q}^{(n)}, \widehat{\boldsymbol{c}}^{(n)}\right) \geq J_{M L}\left(\widehat{\boldsymbol{E}}_{e q}^{(n)}, \widehat{\boldsymbol{c}}^{(n+1)}\right) .
$$

The similar holds true when optimizing $\widehat{\boldsymbol{E}}_{e q}$ based on $\widehat{\boldsymbol{c}}^{(n+1)}$. Namely, since the optimization of $\widehat{\boldsymbol{E}}_{e q}$ is available in closedform, we are guaranteed to find the optimum value of $\widehat{\boldsymbol{E}}_{e q}$ for that particular $\widehat{\boldsymbol{c}}^{(n+1)}$. With that we have

$$
J_{M L}\left(\widehat{\boldsymbol{E}}_{e q}^{(n)}, \widehat{\boldsymbol{c}}^{(n+1)}\right) \geq J_{M L}\left(\widehat{\boldsymbol{E}}_{e q}^{(n+1)}, \widehat{\boldsymbol{c}}^{(n+1)}\right)
$$

By comparing (11) with (12), we see that each iteration of the alternating optimization method either decreases or maintains the associated value in the cost function. This guarantees the convergence of the method, either to the global optimum, a local optimum or a saddle point of $J_{M L}\left(\boldsymbol{E}_{e q}, \boldsymbol{c}\right)$.

\section{NUMERICAL RESULTS}

Here we show some simulation results that motivate the use of the proposed ML estimator to process distributed massive MIMO measurements sets of the nature of (5).

We simulate the case of jointly calibrating three APs, each AP having 32 antennas and 3 possible DFT beams. Moreover, the transmit and receive beamforming codebooks are DFT matrices of size $32 \times 32$ and all APs share such codebooks for transmission and reception. 
We define gains of the transmitters chains, namely $t_{1}, t_{2}$ and $t_{3}$, and the gains of the receivers chains, namely $r_{1}, r_{2}$ and $r_{3}$, as i.i.d. (unit-length) phasors with uniform phase distribution across $[0,2 \pi[$. The non-diagonal entries of the symmetric propagation channel matrix $\boldsymbol{H}_{p}^{i, j} \forall i, j$, are i.i.d. zero-mean unit-variance circularly symmetric complex-valued Gaussian variables, and the non-diagonal entries of the additive receiver noise matrices, $\boldsymbol{N}_{i, j}$ and $\boldsymbol{N}_{j, i}$, are i.i.d. zero-mean circularly symmetric complex-valued Gaussian variables with variance $\sigma^{2}$. (The diagonal entries are undefined as previously mentioned.) The calibration SNR is here defined as $\sigma^{-2}$. We use the ML-based alternating algorithm proposed above to post process the measurements and estimate the calibration matrix $\boldsymbol{C}=\operatorname{diag}\{\boldsymbol{c}\}$, where $\boldsymbol{c}=\left[c_{1}, \ldots c_{M}\right]^{T}$.

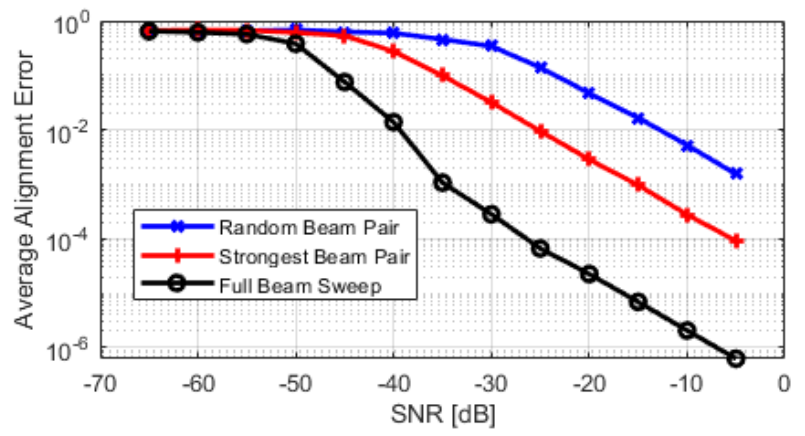

Figure 1: Alignment Error metric versus SNR for the case of calibrating using 3 distinct measurements sets.

Since the main application for the calibration weights is in the context of (reciprocity-based) beamforming (and beamforming is only concerned with complex amplitude differences between antenna elements, and not with their absolute values), any scaled version of a calibration vector is equally good in terms of beamforming performance, i.e. the vector estimate $\hat{\boldsymbol{c}}$ is as good as the vector $\alpha \hat{\boldsymbol{c}}$, for $\alpha$ being any non-zero complex number. Thus, one calibration performance error metric that takes this into account is one minus the squared cosine of the principal angle between the subspace spanned by the true coefficient vector $\boldsymbol{c}$ and the subspace spanned by the vector estimate $\hat{\boldsymbol{c}}$. For example, when the estimate is perfect and the subspace of $\boldsymbol{c}$ is perfectly aligned with the subspace of $\hat{\boldsymbol{c}}$, the error metric equals to zero. This error metric can be written as

$$
\text { Average Alligment Error }=\mathrm{E}\left\{1-\frac{\left|\boldsymbol{c}^{H} \hat{\boldsymbol{c}}\right|^{2}}{\boldsymbol{c}^{H} \boldsymbol{c} \hat{\boldsymbol{c}}^{H} \hat{\boldsymbol{c}}}\right\}
$$

where the expectation operator $\mathrm{E}\{$.$\} acts jointly over the$ random sources of the signal model.
Figure 1 depicts the Average Alignment Error metric versus SNR for the case of calibrating with three distinct measurements sets. Two of the three measurement sets use only one double-directional measurement between each pair of APs in order to compute the calibration coefficients. In this case, state-of-the-art calibration schemes originally designed for fully-digital systems, can be used straightforwardly to postprocess the measurement sets. The third measurement set consists of the case of performing a full-beam sweep for each AP pair, and post-processing the resulting entire measurement with the proposed algorithm (since state-of-the-art methods cannot be used here).

The blue curve represents the case of using only one double directional measurement between each pair of APs, where each beamformer pair (i.e. the transmit and receive beams) is picked at random with equal probability among all possible beamforming pairs. The red curve represents the case of using one double-directional measurement between each pair of APs, where each measured is associated with the beamformer pair that maximizes the energy of the effective channel gain. Finally, the black curve represents the case of combining the measurements from all beam pair combinations, according to the estimator proposed above.

The gap between the blue and red curves is justified by the strongest measurement of the set always yielding equal for greater calibration SNR compared to using an arbitrary beam pair. Finally, the gap between the red and black curves, shows how measurements associated with beam pairs, other than the beam pair yielding the highest link budget, contribute for enhancing the calibration quality. Thus, if measurements other than the measurements associated with the strongest beam pair are available, they should be also post-processed and used for calibration purposes. This motivates the usage of neat signal processing methods, as the proposed algorithm., which is able to post-process multi-beam measurements for calibration - a feature not existing in state-of-the-art massive MIMO calibration literature.

\section{REFERENCES}

Interdonato, G., Björnson, E., Quoc Ngo, H. et al. Ubiquitous cell-free Massive MIMO communications. J Wireless Com Network 2019, 197 (2019).

[2] L. Van der Perre, E. G. Larsson, F. Tufvesson, L. D. Strycker, E. Björnson and O. Edfors, "RadioWeaves for efficient connectivity: analysis and impact of constraints in actual deployments," 2019 53rd Asilomar Conference on Signals, Systems, and Computers, Pacific Grove, CA, USA, 2019, pp. 15-22,

[3] J. Vieira, F. Rusek, O. Edfors, S. Malkowsky, L. Liu, and F. Tufvesson, "Reciprocity calibration for massive MIMO: Proposal, modeling and validation," IEEE Trans. Wireless Commun., vol. 16, no. 5, pp. 3042-3056, May 2017.

[4] R. Rogalin et al., "Scalable synchronization and reciprocity calibration for distributed multiuser MIMO,'” IEEE Trans. Wireless Commun., vol. 13, no. 4, pp. 1815-1831, Apr. 2014.

\section{ACKNOWLEDGEMENTS}

This project has received funding from the European Union's Horizon 2020 research and innovation programme under grant agreement No. 101013425.

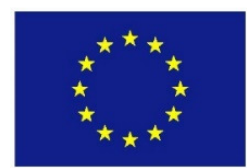

